\title{
Genetic basis and fitness consequences of winglessness in the two-spot ladybird beetle, Adalia bipunctata
}

\author{
H Ueno ${ }^{1,2}$, PW de Jong ${ }^{1,3}$ and PM Brakefield ${ }^{1}$ \\ ${ }^{1}$ Institute of Biology, Leiden University, PO Box 9516, 2300 RA Leiden, The Netherlands; ${ }^{2}$ Laboratory of Biology, Faculty of Education, \\ Niigata University, 8050 Ikarashi-Ni-No-Cho, Niigata 950-2181, Japan; ${ }^{3}$ Laboratory of Entomology, Wageningen University, \\ Binnenhaven 7, Wageningen, PO Box 8031, 6700 EH Wageningen, The Netherlands
}

\begin{abstract}
The genetic basis and fitness consequences of winglessness were investigated in the two-spot ladybird beetle, Adalia bipunctata. By breeding lines from a wingless individual found at The Uithof, Utrecht in The Netherlands, the wingless condition was confirmed to be under the control of a major allele, recessive to the wild type. Wingless individuals, on average, had a longer developmental period, a lower egg production and a shorter lifespan than the wild type with wings, suggesting that the expression of the wingless allele has functionally interrelated gene actions involving a wide range of fitness components. While the wingless allele influences various traits, significant among-family variation in
\end{abstract}

the degree of winglessness suggests that its phenotypic expression is also dependent on the genetic background and modifier loci. Furthermore, there was a consistent pattern of correlation between the degree of winglessness and life history traits; the most extreme wingless individuals showed the lowest fitness while those with more fully developed wings tended to have the highest fitness. This correlation suggests that the modifier genes influence both wing formation and fitness components. The significance of such epistatic effects to the evolution of flightlessness in insects is discussed. Heredity (2004) 93, 283-289. doi:10.1038/sj.hdy.6800502 Published online 16 June 2004

Keywords: wingless gene; Adalia bipunctata; evolution of flightlessness

\section{Introduction}

While evolution of flight was the key innovation in the class Insecta that enhanced the use of spatially and temporally heterogeneous habitats, secondary loss of flight ability has occurred repeatedly within the group (Roff, 1990a; Wagner and Liebherr, 1992). Evolution of flight loss has been most often discussed from the point of view of a trade-off between flight ability and energetic costs associated with producing and maintaining wings and flight muscles. Comparisons of forms able to fly with flightless forms in polymorphic insect species have reported a general increase in fecundity and an earlier age of first reproduction for flightless forms than those capable of flight (Harrison, 1980; Roff, 1986a; Roff and Fairbairn, 1991). Thus, the polymorphic species indicate a phenotypic trade-off between flight ability and reproductive potential.

Genes responsible for winglessness itself, and the genetic background constituting an integrated genetic system for a flightless form are two interrelated, yet different foci of natural selection. Generally, wing morph polymorphism appears to be determined either by a single locus-two allele system, or by the additive action of numerous loci (Den Boer et al, 1980; Harrison, 1980;

Correspondence: H Ueno, Laboratory of Biology, Faculty of Education, Niigata University, 8050 Ikarashi-Ni-No-Cho, Niigata 950-2181, Japan. E-mail: hueno@ed.niigata-u.ac.jp

Received 29 October 2003; accepted 3 April 2004; published online 16 June 2004
Roff, 1986a; Roff and Fairbairn, 1991). In the latter case, high heritabilities for wing morph variation have been reported in quantitative genetic studies, suggesting significant amounts of genetic variation on which natural selection can act (Roff, 1986b, 1990b; Walker, 1987; Mousseau and Roff, 1989; Roff and Bradford, 1996).

How flightlessness molds other traits in life history, morphology and physiology is of relevance to the understanding of the evolution of an integrated system involving the flightless form. Life history traits accrued by the flightless form are often interrelated or reinforcing, including higher fecundity, earlier age at first reproduction and a longer lifespan (Ritchie et al, 1987; Denno et al, 1989). Numerous body shape changes are also associated with the reduction of the proportion of the thorax in flightless forms (Smith, 1964). These pleiotropic effects can be mediated physiologically by endocrine products, such as the titer of juvenile hormone acting as the common link between reproduction and wing formation (Roff, 1986a; Roff and Fairbairn, 1991). A genetic basis of the trade-off between egg production and wing formation has been demonstrated in certain species of cricket by examining the correlated response of fecundity to selection for an increased and a decreased proportion of macroptery (Roff, 1990b), and by an examination using sib-analysis (Roff, 1994; Roff and Bradford, 1996).

The consequence of natural selection on flying and flightless forms, and their coordination with the genetic background can be viewed as two discrete peaks of fitness in the multiple dimensions of the character 
landscape (Wright, 1977). Virtually, all the studies to date that have focused on the fitness difference between flying and flightless morphs are attempts to reveal the present shape and pattern of the character landscape, as molded by selection pressures. However, based on the genotypes in present-day populations that have already undergone the past history of selection, the question of actually how in the process the fitness landscape becomes shaped has been poorly documented. The evolution of novelty, where a fraction of a population can move from a peak to form another, has been a central issue in evolutionary biology (Wade and Goodnight, 1991, 1998). Recent developments in theoretical studies have profoundly changed the view on the importance of the role attributed to the functional gene interaction across different loci in the evolution of novelty (Whitlock et al, 1993; Goodnight, 1987, 1988, 1995; Schlichting and Pigliucci, 1998). However, we still have limited information from empirical studies on the genetic basis of the evolution of flightlessness to complement these theoretical studies.

One crucial aspect of the evolution of flightlessness is that of gene interaction. Once genes responsible for flightlessness have been introduced into a population, whether and how the genes have pleiotropic effects on fitness and how expression of the genes is mediated by the genetic background have important implications for the subsequent process of flightlessness evolution. For example, variation in the gene networks maintained in the population provides a starting point for the response to natural selection, and the degree of variation could limit the course and rate of the subsequent evolution, and perhaps even whether evolution of flightlessness can take place at all. Thus, scrutinizing the nature of pleiotropic and epistatic gene action is a significant step to understanding the initial conditions of the evolution of flightlessness. The present study is a first report on pleiotropic effects of a wingless allele found at very low frequency in a natural population of the two-spot ladybird beetle, Adalia bipunctata (L.). We examine effects on fitness components, and epistatic interactions of the wingless allele with other genes in the genetic background leading to variation in expression of winglessness.

The wingless form of the two-spot ladybird beetle, which lacks elytra and hind wings, has been found occasionally in field populations (Majerus and Kearns, 1989; Marples et al, 1993). Inheritance of winglessness was first reported by breeding lines from a wingless individual found at The Uithof, Utrecht in The Netherlands in 1990 (Marples et al, 1993). They found that winglessness is controlled by an allele with a major phenotypic effect that is recessive to the normal winged one. In the breeding experiment, they also observed variation in the expression of winglessness. However, the stock was lost preventing more detailed research. A very similar wingless phenotype was found again in the same location in 2000, with similar variation in the extent of winglessness confirmed in the descendent generations. One of the major aims of this study is find out whether it is at all feasible that the wingless forms are maintained due to life history trade-offs of wing formation, or recurrent mutations produce similar wingless forms. Also, the study of the inheritance of the winglessness will provide a basis for an evo-devo approach to understand the mechanism involved in the formation of the wings. The present study was performed especially to examine the following issues: (1) the mode of inheritance of the wingless gene, (2) the possible pleiotropic effects of the wingless allele on fitness components, (3) the cause of the variation in the expression of winglessness, and (4) the genetic correlation between the variation and fitness components.

\section{Materials and methods}

A schematic representation of the breeding design is shown in Figure 1. All beetles were reared individually in Petri dishes at $22^{\circ} \mathrm{C}, 16 \mathrm{~L}-8 \mathrm{D}$ photoperiod conditions, with pea aphid, Acyrthosiphon pisum (Harris), provided every other day. These rearing conditions were maintained throughout the experiments. The field-collected wingless individual was a male and copulated only once with a winged virgin female from the same population. Using offspring in the F1 generation, three full-sib pairs were made. Each male was also paired with 10 virgin winged females originating from the same population. To obtain the F3 generation, wingless offspring from the full-sib crosses were mated with a winged individual dammed by the normal winged females. Parents were chosen from different families, so that no pair shared the same male in the F1 generation as a sire. Wingless $\times$ wingless crosses were also made in this generation.

All winged individuals in the F3 generation should be heterozygous if the allele responsible for the trait is

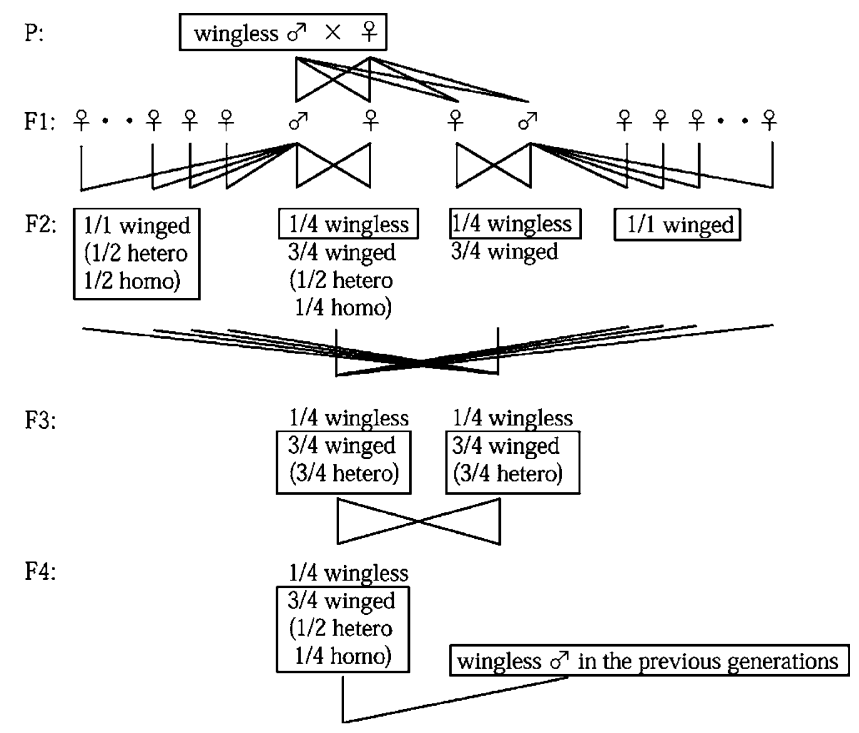

F5:

Figure 1 Schematic representation of the breeding design of the wingless strain. The genotypes and their segregation ratios are those expected if the wingless gene is a major recessive allele. Individuals used to produce the next generation are in the square. The original wingless male shown in the $\mathrm{P}$ generation mated with a winged female, and each male offspring in the F1 generation copulated with one sibling female, as well as with 10 wild-type winged females. Wingless offspring from the full-sib mating were mated with a winged individual dammed by the normal winged females. Winged $x$ winged crosses were made in the F3 generation. Winged females in the F4 were mated with wingless in the previous generations to estimate their genotypes. See further explanation in the text. 
recessive. Winged $\times$ winged crosses were made. Pairs were chosen to reduce inbreeding as far as possible. Offspring from these crosses should be a mixture of the three genotypes in the proportion of 1:2:1. To verify whether winged phenotypes in the F4 generation included both heterozygous and homozygous genotypes with respect to the wild type, winged allele, winged females were backcrossed with wingless males. A female that produced more than five winged offspring and no wingless was judged to be homozygous for the winged allele. Production of any wingless offspring was taken as evidence of the heterozygous genotype.

At the F4 generation from the field collected wingless male, the wingless allele was successfully introduced into different genetic backgrounds. All individuals in the F4 generation were measured for larval period, pupal period and pupal weight. For females, fecundity and longevity were checked every day until the last female died. Females were crossed with wingless males from the fifth day after their eclosion to assess the period of time required for maturation. To equalize the possible effects of copulation on fecundity and longevity for all the females, wingless males were introduced to each female periodically. Winged males were discarded after eclosion.

From the rearing experiment, we scored the following fitness components: developmental traits (larval period, pupal period and pupal weight), reproductive traits (preoviposition period, oviposition period, number of clutches, average clutch size and total fecundity) and longevity after eclosion. We chose total fecundity rather than a measure of effective fecundity that included hatch rate and viability after hatching. We reasoned that hatchability will be influenced not only by female characters but also by male or interactions between the sexes. We believe that in the present context, the effect of the wingless genotype on the total investment to egg production is more important. Furthermore, it is almost practically impossible to follow the viability of all egg clutches at the right timing of hatching (due to sibling cannibalism). For reproductive traits and longevity, only females were measured. Data was ln-transformed before analysis. To determine whether there is any difference between winged and wingless individuals in the developmental traits, an ANOVA was performed for the fitness components with sex, family, wing status and family $\times$ wing status as factors. For reproductive traits and longevity, family and wing status were used as factors. Wing status and sex were regarded as fixed factors, and family and family $\times$ wing status as random factors.

To test for the variation in winglessness among families of the same genotype at the wingless locus, the degree of winglessness was categorized into four classes and the Kruskal-Wallis test was performed with family as a factor. Here the winglessness was categorized as follows: class I, completely wingless, with no elytra and rudimentary hind wings reduced to a very small bud; class II, wingless with elytra reduced to a bud or buds on both outer and medial sides; class III, wingless with half developed elytra; and class IV, wingless with elongated elytra that only lacked the medial development (see also Figure 2). Note here that the continuous variation within each of these categories was not taken into account in subsequent analysis.

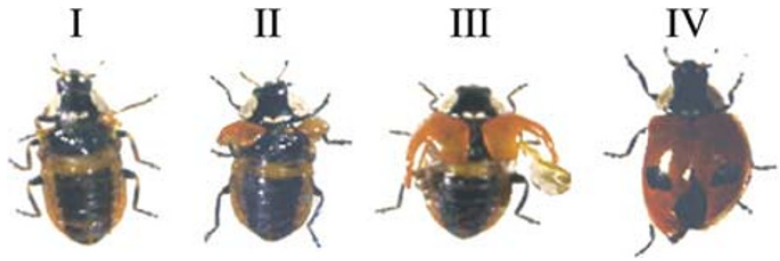

Figure 2 Variation in winglessness categorized to class I, II, III and IV (left to right).

Finally, the correlation between the degree of winglessness and fitness components was checked for larval period, pupal period and pupal weight. An ANOVA was performed with sex, family and the category of winglessness as factors.

\section{Results}

\section{Genetic basis of winglessness}

The overall pattern of inheritance of the wingless phenotype was consistent with the inheritance of a major allele that is recessive to the wild type, winged allele. The details of the breeding experiment were as follows. The female, mated with the original field collected wingless male, produced six viable F1 offspring, three of each sex and all fully winged. The three resulting full-sib pairs making up the F1 generation copulated normally and produced the next generation. All three pairs produced both winged and wingless phenotypes in the F2 generation. The total number of winged and wingless individuals was 89 and 41, respectively, consistent with the $3: 1$ segregation ratio as expected for a single recessive allele $\left(\chi_{1}^{2}=2.48\right.$, NS). There was also no significant difference between the sexes in the proportion of wingless individuals $\left(\chi_{1}^{2}=0.14, N S\right)$. Of 30 winged females mated with F1 males, 27 produced offspring. A total of 56 offspring was obtained, all of which were winged.

Wingless males from one of the three full-sib families copulated normally, but no eggs hatched. This resulted in a reduction of the family number of winged $\times$ wingless crosses in the F2 generation to 15 families and an unbalanced contribution of the families to the next generation. Of the 15 families, seven produced both winged and wingless F3 offspring, and eight produced no wingless. Of 41 offspring produced by the former seven families, 14 were wingless, which is not different from a $1: 1$ ratio $\left(\chi_{1}^{2}=2.27\right.$, NS). There was no evidence for sex-specific expression $\left(\chi_{1}^{2}=1.00, \quad \mathrm{NS}\right)$. Two wingless $\times$ wingless crosses produced 31 and 10 offspring in the F3 generation, respectively, and all of them were wingless.

Winged $\times$ winged crosses in the F3 generation resulted in seven families that produced a substantial number of offspring, and all of them included both phenotypes. In total, 55 wingless were obtained together with 176 winged siblings. There was no deviation from a 3:1 ratio of winged and wingless $\left(\chi_{1}^{2}=0.14, \mathrm{NS}\right)$. Individual family tests detected no family with a significant deviation from the expected 3:1 ratio, and there was no evidence for the heterogeneity of the proportion of winged and wingless individuals among the families $\left(\chi_{6}^{2}=8.46\right.$, NS). The 
proportion of wingless did not differ between the sexes $\left(\chi_{1}^{2}=0.060\right.$, NS). Backcrossing of winged females to wingless males in the F4 generation determined the genotypes of 54 females. All seven families included both heterozygotes and homozygotes.

\section{Fitness consequences of winglessness}

Wingless individuals showed a significantly longer larval and pupal period (Table 1). Wingless individuals can be identified at the pupal stage as the externally visible pupal wing cases are absent. The mortality rate of winged pupae was $4.9 \%$ (nine pupae out of 185 failed to emerge), while that of wingless was $20.3 \%$ (14 out of 69 ). This difference is highly significant using Fisher's exact test $(P<0.001)$.

An ANOVA to compare variation in the reproductive traits and longevity showed a generally lower fitness of wingless individuals compared with their winged siblings (Table 1). Only females were analyzed for the life history traits associated with reproduction and adult longevity. Wingless females showed a significantly shorter oviposition period, fewer ovipositions, smaller clutch sizes and lower total fecundity (Table 1). Longevity after adult eclosion was also significantly shorter for wingless than for their winged siblings, contributing primarily to total fecundity. The preoviposition period, between eclosion and first oviposition, was significantly shorter for wingless individuals (Table 1).

Only a limited number of winged females could be classified as heterozygotes or homozygotes. The resultant small sample size did not allow detection of any statistically significant difference in most cases, but the pattern among the genotypic means was consistent across the traits (Figure 3). The heterozygous individuals suggest dominance effects of the normal winged allele over wingless, and the degree of the dominance $(k)$ ranged from 0.45 to 0.76 , except in the case of pupal weight that suggests complete dominance.

\section{Variation in the degree of winglessness}

Among the wingless individuals, there was a wide variation in the degree of winglessness. Of 41 wingless from the F2 generation, eight were categorized as class I, 20 as class II, seven as class III, and six as class IV. Corresponding numbers in the F3 generation (total $n=13)$ were 1:5:5:2; and in the F4 $(n=55), 4: 19: 32: 0$. Winglessness was significantly variable in expression among families (Kruskal-Wallis $H=6.43$, d.f. $=2$, $P=0.040$ ), and the proportion of this variance attribu-

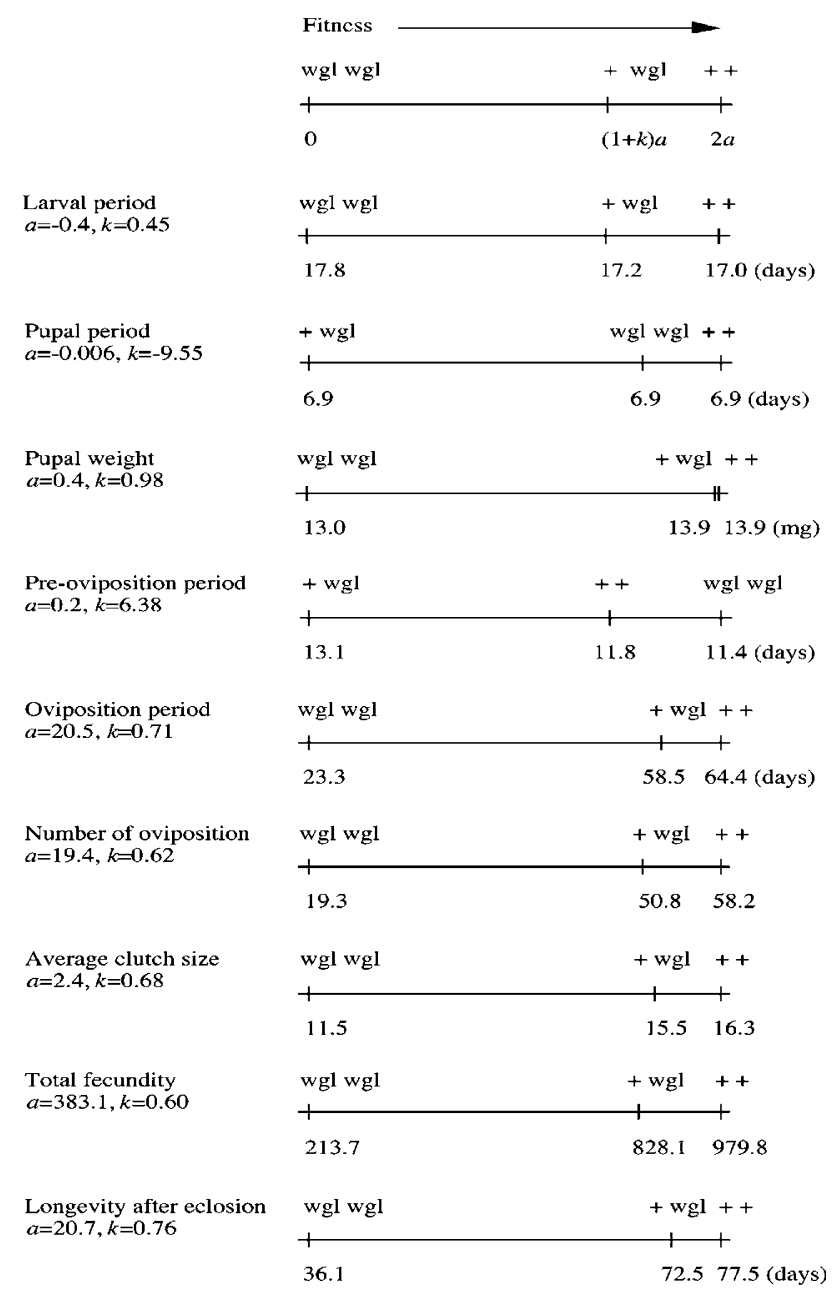

Figure 3 Genotypic value for fitness components with the estimation of the average effect of the winged allele $(a)$ and degree of dominance $(k)$. The homozygous wingless genotype is represented as $\mathrm{wgl} \mathrm{wgl,} \mathrm{heterozygote} \mathrm{as}+\mathrm{wgl}$ and wild-type homozygote as ++ . Fitness increases from left to right.

Table 1 Summary of the results of an ANOVA on developmental traits (larval period, pupal period and pupal weight), reproductive traits (preoviposition period, oviposition period, number of ovipositions, average clutch size and total fecundity) and longevity after adult eclosion

\begin{tabular}{lcrrr}
\hline & Wingless & Winged & d.f. & $F$ \\
\hline Larval period (days) & $17.7 \pm 0.3(55)$ & $17.1 \pm 0.1(176)$ & 1,216 & 4.059 \\
Pupal period (days) & $7.2 \pm 0.1(55)$ & $6.9 \pm 0.04(176)$ & 1,216 & 7.938 \\
Pupal weight (mg) & $12.6 \pm 0.3(55)$ & $12.7 \pm 0.1(176)$ & 1,216 & 0.045 \\
& & & 0.005 \\
Preoviposition period (days) & $11.0 \pm 0.8(21)$ & $12.7 \pm 0.4(95)$ & 1,108 & 5.579 \\
Oviposition period (days) & $25.6 \pm 6.9(21)$ & $56.7 \pm 3.4(95)$ & 1,108 & 18.937 \\
Number of ovipositions & $22.0 \pm 6.1(21)$ & $49.8 \pm 3.0(95)$ & 1,108 & 20.347 \\
Average clutch size & $12.5 \pm 1.1(21)$ & $15.1 \pm 0.5(95)$ & 1,108 & 5.390 \\
Total fecundity & $283.3 \pm 116.1(21)$ & $809.9 \pm 56.5(95)$ & 1,108 & 21.577 \\
& & & $<0.001$ \\
Longevity after eclosion (days) & $37.9 \pm 6.9(21)$ & $70.5 \pm 3.4(95)$ & 1,108 & 0.001 \\
& & & 25.520
\end{tabular}

Adjusted least means for wingless and winged are presented with standard errors. Sample sizes are indicated in parentheses. For developmental traits, an ANOVA was performed with sex, family, wing status, family $\times$ wing status as factors. No significant family $\times$ wing status interaction was detected (not shown). For reproductive traits and longevity, an ANOVA was performed with family and wing status as factors. 

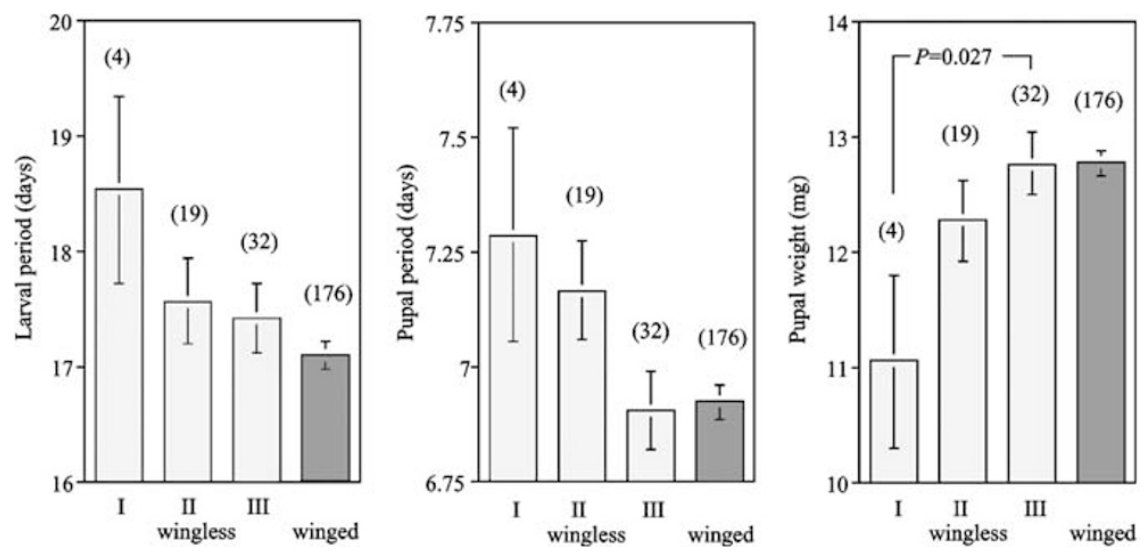

Figure 4 Larval period, pupal period and pupal weight for winged and three categories of wingless individuals. The bar indicates standard errors and the sample size is in parentheses.

table to the family was estimated as 0.317 from the broad-sense heritability for the expression of the wingless gene using variance components calculated from an analysis of variance (family effect in ANOVA, $\left.\mathrm{F}_{6,47}=2.38, P=0.043\right)$.

\section{Correlation between the degree of winglessness and fitness components}

Owing to the small sample size, only developmental traits were tested for the fitness consequences of the degree of winglessness in an ANOVA that excluded the effect of families. The ANOVA detected large and significant among-family variance for both larval period and pupal weight $\left(\mathrm{F}_{6,45}=7.982, \quad P<0.001\right.$ and $\mathrm{F}_{6,45}=3.408, P<0.01$, respectively), but not for pupal period $\left(\mathrm{F}_{6,45}=2.007, P=0.084\right)$. There was a consistent pattern for the correlation between the degree of winglessness and developmental traits (Figure 4). The most extreme wingless individuals showed the lowest fitness while those wingless individuals with the most fully developed wings had the highest fitness, comparable to the winged genotype. The effect of winglessness category was statistically significant for pupal weight $\left(\mathrm{F}_{2,45}=4.567, P=0.016\right)$ and a significant difference was also detected in this trait between category I and III $(P=0.027)$. However, statistical tests were nonsignificant for both larval period and pupal period $\left(\mathrm{F}_{2,45}=0.954\right.$ and 1.002 , respectively).

\section{Discussion}

The results of our breeding experiments indicate complex interactions among genes involved in the expression of winglessness. Figure 5 is a schematic representation of the possible routes of gene interaction. The patterns and proportions of winged and wingless produced over the five generations of this study are consistent with the hypothesis that the wingless trait in our line established from a single wild-collected individual is controlled by a major allele recessive to the wild type. At the same time, it is evident that the gene has strong negative pleiotropic effects on fitness components, suggesting that the expression of the wingless gene has functionally interrelated gene actions with a wide range of key life history

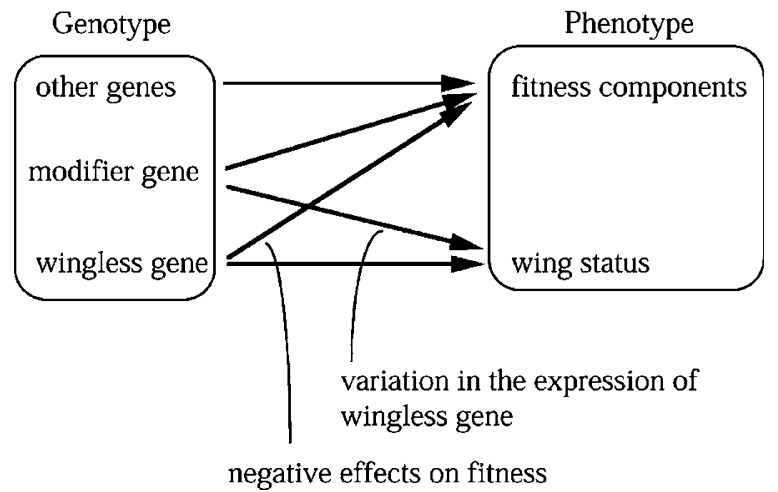

Figure 5 Schematic representation of the possible gene interactions involved in wing formation.

traits involving developmental period, production of eggs and longevity.

It is possible that the effects on reproductive traits and longevity expressed at the adult stage are a direct consequence of being wingless. Wingless beetles may be more vulnerable to environmental stress as they lack elytral coverage over the dorsal surface. On the other hand, the phenotypic effects on life history traits could result from pleiotropy or epistatic genetic effects during preadult development. The data for known heterozygotes at the wingless locus indicate a decline in fitness relative to the homozygote wild type. Thus, the results indicate the multiple effects of the wingless allele across several life history traits.

The trends found in the relationship between the degree of winglessness and developmental traits further support a functional link between wing formation and life history traits. Thus, wingless individuals with more extreme winglessness were associated with a longer developmental period and a smaller pupal weight. Although we have no physiological information about the winglessness in $A$. bipunctata, endocrine signals may affect both wing formation and life history traits. Juvenile hormone, for example, that acts as a basic regulator of insect development, may also be involved in wing formation (cf in the cricket, Gryllus rubens; Zera and Tiebel, 1988, 1989). The physiological and developmental 
bases of winglessness in A. bipunctata will be the subject of further studies.

The preoviposition period is the only trait that indicated a significant difference that could favor the wingless form; these started to lay eggs earlier than winged individuals. However, all the other traits indicated that there is no fitness gain through the suppression of wing formation. The general reduction in fitness components indicates that wingless homozygotes occasionally produced by chance in the field will be simply selected out of the population. The wingless allele in the Utrecht population has presumably been maintained mainly in the form of the heterozygous genotype as a balance between recurrent mutation and selection.

While the wingless allele in $A$. bipunctata may influence various traits, significant among-family variation in the degree of winglessness suggests that its expression is also dependent on the genetic background The correlation between the degree of winglessness and life history traits suggests that genes at other loci modify both wing formation and fitness components. Such epistatic interactions may have a significant evolutionary importance as they may provide the potential for adaptive evolution of the wingless state. Evolution of modifier genes may lead to a co-adapted genetic constitution that neutralizes the deleterious effects of the major mutation (Wright, 1977). Random genetic drift could play a role in such a process when the population is deme-structured. If the process of genetic drift occurs independently in many demes, then in some deme, sometimes, the negative pleiotropic effects of the wingless allele may be counteracted by a chance improvement of the interaction among all the genes. The whole complex, the major wingless mutation and the array of modifiers, may then eventually result in the evolution of a selective advantage to the wingless condition, or a balanced genetic polymorphism, in a particular environment.

Differences in the genetic basis of flightlessness have been demonstrated in studies using wing polymorphic species (Roff, 1986a; Roff and Fairbairn, 1991). In some cases, there is a genetic polymorphism at a single locus, in others, additive control involving a polygenic system. Our preliminary studies in A. bipunctata suggest that these different modes of genetic control of wing formation might be bridged by a process involving the fixation of a major allele in a local deme with subsequent elaboration of the interactions with other modifier genes each of small phenotypic effect. The significant variation in the extent of winglessness among families suggests the possibility of selection for specific modes of expression of winglessness by molding certain background genotypes and altering epistatic interactions. If selection favors longer-winged wingless individuals, as might be predicted from observations in the present study, wingless individuals with almost perfectly formed elytra in which wing formation is nearly normal could then result.

A similar situation is suggested by the variation for the genetic basis of wing polymorphism found in closely related carabid beetles. This polymorphism is controlled by two major alleles at a single locus in one species, while in a related species wing formation has a genetic component but is not a simple Mendelian trait (Aukema, 1990). The latter pattern, found in several other insect species (McFarlane, 1964; Rose, 1972; Harrison, 1979), could be interpreted as major gene control for wing formation with polygenic variation in a threshold response, thus indicating the presence of the intermediate or transient genetic system between major gene control and polygenic system in natural populations. The relative importance of the effect of the major gene for winglessness and such modifier genes is likely to vary from species to species, from population to population, and from gene to gene. The wingless gene and its variation in expression in A. bipunctata should, however, provide us with one welcome opportunity to trace the initial conditions in the evolution of wing polymorphisms and the wingless state in insects.

\section{Acknowledgements}

We are indebted to Ayako Ueno for helping us to collect beetles and actually finding the original wingless individual. We would like to express our sincere thanks to Els Schlatmann, Niels Wurzer, Mariel Lavrijsen and Bert de Winter for cultivating bean plants, and Edda Japing and Kees Koops for help in rearing wingless beetles.

\section{References}

Aukema B (1990). Wing-length determination in two wingdimorphic Calathus species (Coleoptera: Carabidae). Hereditas 113: 189-202.

Den Boer PJ, Van Huizen THP, Den Boer-Daanje W, Aukema B, Den Bieman CFM (1980). Wing polymorphism and dimorphism in ground beetles as stages in an evolutionary process (Coleoptera: Carabidae). Entomol Gen 6: 107-134.

Denno RF, Olmstead KL, McCloud ES (1989). Reproductive cost of flight capability: a comparison of life history traits in wing dimorphic planthoppers. Ecol Entomol 14: 31-44.

Goodnight CJ (1987). On the effect of founder events on epistatic genetic variation. Evolution 41: 80-91.

Goodnight CJ (1988). Population differentiation and the transmission of density effects between generations. Evolution 42: 399-403.

Goodnight CJ (1995). Epistasis and the increase in additive genetic variance: implications for phase I of Wright's shifting balance process. Evolution 49: 501-511.

Harrison RG (1979). Flight polymorphisms in the field cricket Gryllus pennsylvanicus. Oecologia 40: 125-132.

Harrison RG (1980). Dispersal polymorphisms in insects. Ann Rev Ecol Systems 11: 95-118.

Majerus M, Kearns P (1989). Ladybirds. The Richmond Publishing Co. Ltd: Slough.

Marples NM, de Jong PW, Ottenheim MM, Verhoog MD, Brakefield PM (1993). The inheritance of a wingless character in the 2spot ladybird (Adalia bipunctata). Entomol Exp Appl 69: 69-73.

McFarlane JE (1964). Factors affecting growth and wing polymorphism in Gryllodes sigillatus (Walk.): dietary protein level and a possible effect of photoperiod. Can J Zool 42: 767-771.

Mousseau TA, Roff DA (1989). Geographic variability in the incidence and heritability of wing dimorphism in the striped ground cricket, Allonemobius fasciatus. Heredity 62: 315-318.

Ritchie MG, Butlin RK, Hewitt GM (1987). Causation, fitness effects and morphology of macropterism in Chorthippus parallelus (Orthoptera: Acrididae). Ecol Entomol 12: 209-218.

Roff DA (1986a). The evolution of wing dimorphism in insects. Evolution 40: 1009-1020.

Roff DA (1986b). The genetic basis of wing dimorphism in the sand cricket, Gryllus firmus and its relevance to the evolution of wing dimorphism in insects. Heredity 57: 221-231. 
Roff DA (1990a). The evolution of flightlessness in insects. Ecol Monogr 60: 389-421.

Roff DA (1990b). Antagonistic pleiotropy and the evolution of wing dimorphism in the sand cricket, Gryllus firmus. Heredity 65: 169-177.

Roff DA (1994). Evidence that the magnitude of the trade-off in a dichotomous trait is frequency dependent. Evolution 48: $1650-1656$

Roff DA, Bradford MJ (1996). Quantitative genetics of the tradeoff between fecundity and wing dimorphism in the cricket Allnemobius socius. Heredity 76: 178-285.

Roff DA, Fairbairn DJ (1991). Wing dimorphisms and the evolution of migratory polymorphisms among the insects. Am Zool 31: 243-251.

Rose DJW (1972). Dispersal and quality in populations of Cicadulina species (Cicadellidae). J Anim Ecol 41: 589-609.

Schlichting CD, Pigliucci M (1998). Phenotypic Evolution: A Reaction Norm Perspective. Sinauer Associates, Inc.: Sunderland, MA.

Smith DS (1964). The structure and development of flightless Coleoptera: a light and electron microscopic study of the wings, thoracic exoskelton and rudimentary flight musculature. J Morphol 114: 107-184.

Wade MJ, Goodnight CJ (1991). Wright's shifting balance theory: an experimental study. Science 253: 1015-1018.

Wade MJ, Goodnight CJ (1998). Perspective: the theories of Fisher and Wright in the context of metapopulations: when nature does many small experiments. Evolution 52 1537-1553.

Wagner DL, Liebherr JK (1992). Flightlessness in insects. TREE 7: 216-220.

Walker TJ (1987). Wing dimorphism in Gryllus rubens (Orthoptera: Gryllidae). Ann Entomol Soc Am 80: 547-560.

Whitlock MC, Phillips PC, Wade MJ (1993). Gene interaction affects the additive genetic variance in subdivided populations with migration and extinction. Evolution 47: 1758-1769.

Wright S (1977). Evolution and the Genetics of Populations, Vol. 3. Experimental Results and Evolutionary Deductions. University of Chicago Press: Chicago.

Zera AJ, Tiebel KC (1988). Brachytpterizing effect of group rearing, juvenile hormone-III and methoprene on wing length development in the wing-dimorphic cricket, Gryllus rubens. J Insect Physiol 34: 489-498.

Zera AJ, Tiebel KC (1989). Differences in juvenile hormone esterase activity between presumptive macropterous and brachypterous Gryllus rubens: implications to the hormonal control of wing polymorphism. J Insect Physiol 35: 7-18. 